\title{
Majorization and Coefficient Problems for a General Class of Starlike Functions
}

\author{
Nak Eun Cho ${ }^{1}$ (D), Zahra Oroujy ${ }^{2}$, Ebrahim Analouei Adegani ${ }^{2, *(D)}$ and Ali Ebadian ${ }^{2}$ \\ 1 Department of Applied Mathematics, Pukyong National University, Busan 608-737, Korea; \\ necho@pknu.ac.kr \\ 2 Department of Mathematics, Urmia University, Urmia 57135-165, Iran; z.orouji@urmia.ac.ir (Z.O.); \\ a.ebadian@urmia.ac.ir (A.E.) \\ * Correspondence: analoey.ebrahim@gmail.com
}

Received: 17 February 2020; Accepted: 3 March 2020; Published: 18 March 2020

\begin{abstract}
In the current paper, we study a majorization issue for a general category $\mathcal{S}^{*}(\vartheta)$ of starlike functions, the region of which is often symmetric with respect to the real axis. For various special symmetric functions $\vartheta$, corresponding consequences of the main result are also presented with some relevant connections of the outcomes rendered here with those obtained in recent research. Moreover, coefficient bounds for some majorized functions are estimated.
\end{abstract}

Keywords: subordination; majorization; starlike function

MSC: Primary 30C45; Secondary 30C80

\section{Introduction and Preliminaries}

Let $\mathbb{U}$ denote the unit disk $\{z \in \mathbb{C}:|z|<1\}$ and $\mathcal{H}$ represent the class of analytic functions in $\mathbb{U}$. We denote by $\mathcal{A}$ the subclass of $\mathcal{H}$ consisting of functions

$$
f(z)=z+\sum_{n=2}^{\infty} a_{n} z^{n} .
$$

Let $\Phi$ represent the category of all analytic functions $\omega$ in $\mathbb{U}$ that satisfy the requirements of $\omega(0)=0$ and $|\omega(z)|<1$ for $z \in \mathbb{U}$, i.e., we consider $\Phi$ the set of Schwarz functions.

Definition 1. [1,2] For two analytic functions $\theta$ and $\Theta$ in the unit disk, we state $\theta(z)$ is quasi-subordinate to $\Theta(z)$ if there is a function $v(z)$, analytic in $\mathbb{U}$, so that $\theta(z) / v(z)$ is analytic in $\mathbb{U}$

$$
\frac{\theta(z)}{v(z)} \prec \Theta(z) \quad(z \in \mathbb{U})
$$

and $|v(z)| \leq 1(z \in \mathbb{U})$, where $\prec$ stands for the usual subordination for analytic functions in $\mathbb{U}$. We denote the above quasi-subordination by

$$
\theta(z) \prec_{q} \Theta(z) \quad(z \in \mathbb{U})
$$

It is remarkable that the relation (2) can be rewritten as follows

$$
\theta(z)=v(z) \Theta(\omega(z)) \quad(z \in \mathbb{U})
$$


where $|v(z)| \leq 1(z \in \mathbb{U})$ and $\omega \in \Phi$. For $v(z) \equiv 1$ and $\omega(z)=z$, the quasi-subordination reduces the subordination [3] and the majorization [4], i.e.,

$$
\theta(z)=\Theta(\omega(z)) \quad(z \in \mathbb{U})
$$

written as $\theta(z) \prec \Theta(z)$ and

$$
\theta(z)=v(z) \Theta(z) \quad(z \in \mathbb{U})
$$

written as $\theta(z) \ll \Theta(z)$, respectively.

Using the principle of subordination, a different subclass $\mathcal{S}^{*}(\vartheta)$ of starlike functions was defined by Ma and Minda [5] where $\vartheta$ is analytic and univalent with $\operatorname{Re}(\vartheta(z))>0$ in $\mathbb{U}$, starlike with $\vartheta(0)=1$ and $\vartheta(\mathbb{U})$ is symmetric with respect to the real axis so that $\vartheta^{\prime}(0)>0$. They introduced the class by:

$$
\mathcal{S}^{*}(\vartheta):=\left\{f \in \mathcal{A}: \frac{z f^{\prime}(z)}{f(z)} \prec \vartheta(z), z \in \mathbb{U}\right\} .
$$

For example, for the function $\vartheta(z)=(1+C z) /(1+D z)(-1 \leq D<C \leq 1)$, the class $\mathcal{S}^{*}(\vartheta)$ becomes the subclass $\mathcal{S}^{*}[C, D]$ of the well-known Janowski starlike functions. By replacing $C=1-2 \gamma$ and $D=-1$ where $0 \leq \gamma<1$, we obtain the category $\mathcal{S}^{*}(\gamma)$ of the starlike functions of order $\gamma$. Specifically, $\mathcal{S}^{*}:=\mathcal{S}^{*}(0)$ is the well-known category of starlike functions in $\mathbb{U}$. Some special subclasses of the class $\mathcal{S}^{*}(\vartheta)$ play a significant act in geometric function theory because of their geometric properties. It is fairly common that a function in one of these subclasses is lying in a given region in the right half-plan and the region is often symmetric with respect to the real axis.

Taking $\vartheta(z)=\sqrt{1+z}$ we get a category of $\mathcal{S}_{L}^{*}$, which was reviewed by Sokół and Stankiewicz [6] and implies that $f \in \mathcal{S}_{L}^{*}$ if and only if $z f^{\prime}(z) / f(z) \in \mathrm{B}$, where $\mathrm{B}=\left\{w \in \mathbb{C}:\left|w^{2}-1\right|<1\right\}$. Moreover, the features of the category $\mathcal{S}_{e}^{*}:=\mathcal{S}^{*}\left(e^{z}\right)$ comprising functions $f \in \mathcal{A}$, with the requirement of $\left|\log \left(z f^{\prime}(z) / f(z)\right)\right|<1$ was considered by Mendiratta et al. in [7]. In [8] researchers investigated the category $\mathcal{S}^{*}(h)$, where

$$
h(z)=z+\sqrt{1+z^{2}}=1+z+\frac{z^{2}}{2}+\cdots,
$$

and proved that $f \in \mathcal{S}^{*}(h)$ if and only if $z f^{\prime}(z) / f(z) \in \mathrm{R}$, where $\mathrm{R}=\left\{w \in \mathbb{C}:\left|w^{2}-1\right|<2|w|\right\}$. Lately, Kanas et al. [9] defined the class $\mathcal{S} \mathcal{T}_{h p l}(b):=\mathcal{S}^{*}\left(\mathfrak{q}_{b}(z)\right)$ and obtained some geometric properties in this class where the function

$$
\mathfrak{q}_{b}(z)=\frac{1}{(1-z)^{b}}=\mathrm{e}^{b \log (1-z)}=1+b z+\frac{b(b+1)}{2} z^{2}+\frac{b(b+1)(b+2)}{6} z^{3}+\cdots \quad(0<b \leq 1),
$$

where the branch of the logarithm is considered by $\mathfrak{q}_{b}(0)=1$, maps $\mathbb{U}$ onto a region, which is bounded by a right branch of a hyperbola

$$
\mathbb{H}(b)=\left\{\sigma \mathrm{e}^{\mathrm{i} \chi}: \sigma=\frac{1}{(2 \cos (\chi / b))^{b}},|\chi|<\frac{\pi b}{2}\right\} .
$$

Moreover, $\mathfrak{q}_{b}(\mathbb{U})$ is symmetric about the real axis, starlike with respect to $\mathfrak{q}_{b}(0)=1$ and convex. Further $\mathfrak{q}_{b}(z)$ has positive real part in $\mathbb{U}$ and $\mathfrak{q}_{b}^{\prime}(0)>0$. Therefore, $\mathfrak{q}_{b}(z)$ satisfies the classification of Ma-Minda functions.

Recently, Goel and Kumar [10] introduced the class $\mathcal{S}_{S J}^{*}$ and obtained some different problems in this class as follows:

$$
\mathcal{S}_{S J}^{*}=: \mathcal{S}^{*}(J)=\left\{f \in \mathcal{A}: \frac{z f^{\prime}(z)}{f(z)} \prec \frac{2}{1+\mathrm{e}^{-z}}, z \in \mathbb{U}\right\}
$$


The modified sigmoid function

$$
J(z)=\frac{2}{1+\mathrm{e}^{-z}}=1+\frac{1}{2} z-\frac{1}{24} z^{3}+\cdots,
$$

maps $\mathbb{U}$ onto a domain $\Delta_{S J}:=\{\xi \in \mathbb{C}:|\log (\xi /(2-\xi))|<1\}$, which is symmetric about the real axis. Also, $J(z)$ is a convex function and so starlike function with respect to $J(0)=1$. Moreover, $J(z)$ has positive real part in $\mathbb{U}$ and $J^{\prime}(0)>0$. Therefore, $J(z)$ satisfies the classification of Ma-Minda functions.

MacGregor [4] and Altintas et al. [11] (see also [12]) studied the majorization issues for the category $\mathcal{S}^{*}$ and for specific analytic functions by convex and starlike functions of complex order.

Theorem 1. ([4], Theorem 1. A) Let $\theta(z)$ and $\Theta(z)$ be analytic functions in $\mathbb{U}$ with $\theta(z) \ll \Theta(z)$ and $\Theta(0)=0$. If $0 \leq r \leq \sqrt{2}-1$, then

$$
\max _{|z|=r}\left|\theta^{\prime}(z)\right| \leq \max _{|z|=r}\left|\Theta^{\prime}(z)\right| .
$$

By setting $\Theta(z)=z$, in above outcome we conclude the next well-known result:

Lemma 1. [13] If $\theta(z)$ be analytic in $\mathbb{U}$ with $|\theta(z)| \leq 1$ and $\theta(0)=0$, then $\left|\theta^{\prime}(z)\right| \leq 1$ for $|z| \leq \sqrt{2}-1$.

Recently, several authors have investigated majorization issues for the families of meromorphic and multivalent meromorphic or univalent and multivalent functions including various linear and nonlinear operators, which all are subordinated by the similar function $\vartheta(z)=(1+C z) /(1+D z)$ (for example, see [14-20]). Lately, Tang et al. [21] studied majorization problem for the subclasses of $\mathcal{S}^{*}(\vartheta)$, which are relevant to $\mathcal{S}^{*}(1+\sin z)$ and $\mathcal{S}^{*}(\cos z)$, regardless of any linear or nonlinear operators. Hence, in this work, we study a majorization issue for the general category $\mathcal{S}^{*}(\vartheta)$ with various special consequences of the main result. Also, some suitable relations of the outcomes are presented with those reported in the earlier results. Moreover, coefficient estimates for majorized functions related to the class $\mathcal{S}^{*}(\vartheta)$ are obtained.

\section{Main Results}

We first state and establish a majorization feature for the general category $\mathcal{S}^{*}(\vartheta)$ and then some consequences of the main result are stated.

Theorem 2. Let $\theta \in \mathcal{A}, \Theta \in \mathcal{S}^{*}(\vartheta)$ with $\theta(z) \ll \Theta(z)$, then $\left|\theta^{\prime}(z)\right| \leq\left|\Theta^{\prime}(z)\right|$ for all $z$ in the disk $|z| \leq r_{1}$, where $r_{1}$ is the smallest positive root of the equation

$$
\min _{|z|=r}|\vartheta(z)|\left(1-r^{2}\right)-2 r=0, \quad r \in(0,1)
$$

Proof. Since $\theta(z) \ll \Theta(z)$, considering the concept of majorization, there is a function $v$ that is analytic in $\mathbb{U}$ with $|v(z)| \leq 1$ satisfying

$$
\theta(z)=v(z) \Theta(z)
$$

Differentiating the last equality with respect to $z$, it follows that

$$
\theta^{\prime}(z)=v^{\prime}(z) \Theta(z)+v(z) \Theta^{\prime}(z)=\Theta^{\prime}(z)\left(v^{\prime}(z) \frac{\Theta(z)}{\Theta^{\prime}(z)}+v(z)\right) .
$$


Now, let $\Theta \in \mathcal{S}^{*}(\vartheta)$, then from the subordination concept, there exists a $\omega \in \Phi$ with $|\varrho(z)| \leq$ $|z|=r$ so that

$$
\frac{z \Theta^{\prime}(z)}{\Theta(z)}=\vartheta(\omega(z))
$$

or equivalently

$$
\frac{\Theta(z)}{\Theta^{\prime}(z)}=\frac{z}{\vartheta(\omega(z))}
$$

Since $\operatorname{Re}(\vartheta(z))>0$ in $\mathbb{U}$, so $\vartheta(z) \neq 0$ for all $z \in \mathbb{U}$. Now, by the minimum modulus principle we conclude

$$
\min _{|z|=r}|\vartheta(z)| \leq \min _{|\omega(z)|=r} \mid \vartheta\left((\omega(z))\left|=\min _{|\omega(z)| \leq r}\right| \vartheta((\omega(z)) \mid .\right.
$$

We know that $\vartheta$ is a continuous function with $\operatorname{Re}(\vartheta(z))>0$ in $\mathbb{U}$ and so $\min _{|z|=r}|\vartheta(z)| \neq 0$. Therefore, from this point, (4) and the above relation we obtain

$$
\left|\frac{\Theta(z)}{\Theta^{\prime}(z)}\right|=\frac{|z|}{|\vartheta(\omega(z))|} \leq \frac{r}{\min _{|z|=r}|\vartheta(z)|} .
$$

On the other hand, applying the popular inequality for Schwarz functions, which states that

$$
\left|v^{\prime}(z)\right|\left(1-|z|^{2}\right) \leq 1-|v(z)|^{2} .
$$

Utilizing (5) and (6) in (3), we obtain

$$
\left|\theta^{\prime}(z)\right| \leq\left(\frac{1-|v(z)|^{2}}{1-|z|^{2}} \frac{r}{\min _{|z|=r}|\vartheta(z)|}+|v(z)|\right)\left|\Theta^{\prime}(z)\right| \quad(|z|=r<1) .
$$

Setting $|v(z)|=\gamma(0 \leq \gamma \leq 1)$, it follows that

$$
\left|\theta^{\prime}(z)\right| \leq\left(\frac{1-\gamma^{2}}{1-r^{2}} \frac{r}{\min _{|z|=r}|\vartheta(z)|}+\gamma\right)\left|\Theta^{\prime}(z)\right| \quad(0 \leq \gamma \leq 1) .
$$

Define

$$
l(r, \gamma)=\gamma+\frac{1-\gamma^{2}}{1-r^{2}} \frac{r}{\min _{|z|=r}|\vartheta(z)|} \quad(0 \leq \gamma \leq 1,0<r<1) .
$$

In order to determine $r_{1}$, we must choose

$$
r_{1}=\max \{r \in[0,1): l(r, \gamma) \leq 1, \gamma \in[0,1]\} .
$$

We know $l(r, \gamma) \leq 1$ if and only if

$$
0 \leq \min _{|z|=r}|\vartheta(z)|\left(1-r^{2}\right)-(1+\gamma) r=: p(r, \gamma) .
$$

Clearly, the function $p(r, \gamma)$ chooses its minimum value for $\gamma=1$, that is,

$$
\min \{p(r, \gamma): \gamma \in[0,1]\}=p(r, 1)=: p(r),
$$

where

$$
p(r)=\min _{|z|=r}|\vartheta(z)|\left(1-r^{2}\right)-2 r \quad(0<r<1) .
$$


Further, since $p(0)=1>0$ and $p(1)=-2<0$, there exists $r_{1}$, so that for all $r \in\left[0, r_{1}\right]$, we have $p(r) \geq 0$ where $r_{1}$ is the smallest positive root of the above equality and this completes the proof.

Remark 1. Since $\vartheta$ is a convex and symmetric with $\operatorname{Re}(\vartheta(z))>0$, we get $\min _{|z|=r}|\vartheta(z)|=\vartheta(-r)$ (see [22], Proposition 5.3).

The following corollary concludes a majorization property for the subclass $\mathcal{S} \mathcal{T}_{h p l}(b)$ considering Lemma 2.1 in [9].

Corollary 1. Let $\theta \in \mathcal{A}, \Theta \in \mathcal{S T}_{\text {hpl }}(b)$ with $\theta(z) \ll \Theta(z)$. Then for all $z$ in the disk $|z| \leq r_{2}$, we get $\left|\theta^{\prime}(z)\right| \leq\left|\Theta^{\prime}(z)\right|$, where $r_{2}$ is the smallest positive root of the equation

$$
1-r^{2}-2 r(1+r)^{b}=0, \quad r \in(0,1) .
$$

Example 1. If we choose the functions

$$
\Theta(z)=z \exp \left(B_{1} z\right) \in \mathcal{S} \mathcal{T}_{h p l}(b) \text { for } 0<B_{1}<1-2^{-b}
$$

(see [9]) and

$$
\theta(z)=\frac{z}{3+z} \exp \left(B_{1} z\right)
$$

then these functions satisfy in the relation $\theta(z) \ll \Theta(z)$ with $v(z)=\frac{1}{3+z}$. Therefore, from Corollary 1 we have

$$
\left|\frac{3}{(3+z)^{2}}+\frac{B_{1} z}{3+z}\right| \leq\left|1+B_{1} z\right|
$$

for $|z| \leq r_{2}$.

Since $2 /\left(1+e^{r}\right) \leq 2 /\left|1+e^{-z}\right|(|z|=r<1)$, the next corollary concludes a majorization feature for the subclass $\mathcal{S}_{S J}^{*}$.

Corollary 2. Let $\theta \in \mathcal{A}, \Theta \in \mathcal{S}_{S J}^{*}$ with $\theta(z) \ll \Theta(z)$. Then $\left|\theta^{\prime}(z)\right| \leq\left|\Theta^{\prime}(z)\right|$ for $|z| \leq r_{3}$, where $r_{3}$ is the smallest positive root of the equation

$$
\frac{2}{1+e^{r}}\left(1-r^{2}\right)-2 r=0, \quad r \in(0,1)
$$

Since

$$
|\sin z| \leq \sinh r \quad(|z|=r<1)
$$

(see [23]), we have

$$
0<1-\sinh r \leq 1-|\sin z| \leq|1+\sin z| \quad(|z|=r<0.8813735870),
$$

so the following corollary concludes a majorization property for the subclass $\mathcal{S}_{s}^{*}:=\mathcal{S}^{*}(1+\sin z)$ studied by Cho et al. in [23] and also we have the result which was given by Tang et al. in ([20], Theorem 2.1).

Corollary 3. Let $\theta \in \mathcal{A}, \Theta \in \mathcal{S}_{s}^{*}$ with $\theta(z) \ll \Theta(z)$. Then for $|z| \leq r_{4}$, we get $\left|\theta^{\prime}(z)\right| \leq\left|\Theta^{\prime}(z)\right|$, where $r_{4}$ is the smallest positive root of the equation

$$
\left(1-r^{2}\right)(1-\sinh r)-2 r=0, \quad r \in(0,1) .
$$


Example 2. If we consider the functions

$$
\Theta(z)=z \mathrm{e}^{z / 2} \in \mathcal{S}_{S}^{*}
$$

(see [23]) and

$$
\theta(z)=\frac{z}{2+z} \mathrm{e}^{z / 2},
$$

then we have $\theta(z) \ll \Theta(z)$ with $v(z)=\frac{1}{2+z}$. Therefore, from Corollary 3 we get

$$
\left|\frac{2}{(2+z)^{2}}+\frac{z}{2(2+z)}\right| \leq\left|1+\frac{z}{2}\right|
$$

for $|z| \leq r_{4}$.

Since

$$
\cos r \leq|\cos z| \quad(|z|=r<1),
$$

the following corollary concludes a majorization property for a subclass $\mathcal{S}^{*}(\cos z)$ and also we have a correction of the result which was given by Tang et al. in ([21], Theorem 2.2).

Corollary 4. Let $\theta \in \mathcal{A}, \Theta \in \mathcal{S}^{*}(\cos z)$ with $\theta(z) \ll \Theta(z)$. Then $\left|\theta^{\prime}(z)\right| \leq\left|\Theta^{\prime}(z)\right|$ for $|z| \leq r_{5}$, where $r_{5}$ is the smallest positive root of the equation

$$
\left(1-r^{2}\right) \cos r-2 r=0, \quad r \in(0,1) .
$$

In the following corollaries, we obtain majorization properties for two subclasses $\mathcal{S}_{\alpha, \mathrm{e}}^{*}=\mathcal{S}^{*}(\alpha+$ $\left.(1-\alpha) \mathrm{e}^{z}\right)(0 \leq \alpha<1)$ and $\mathcal{S} \mathcal{L}^{*}(\alpha)=\mathcal{S}^{*}(\alpha+(1-\alpha) \sqrt{1+z})(0 \leq \alpha<1)$, which were defined by Khatter et al. considering Lemma 2.1 in [24]. For $\alpha=0$, these results reduce to the subclasses $\mathcal{S}^{*}\left(\mathrm{e}^{z}\right)$ and $\mathcal{S}^{*}(\sqrt{1+z})$ (see $\left.[6,7]\right)$.

Corollary 5. Let $\theta \in \mathcal{A}, \Theta \in \mathcal{S}_{\alpha, \mathrm{e}}^{*}$ with $\theta(z) \ll \Theta(z)$. Then $\left|\theta^{\prime}(z)\right| \leq\left|\Theta^{\prime}(z)\right|$ for $|z| \leq r_{6}$, where $r_{6}$ is the smallest positive root of the equation

$$
\left[\alpha+(1-\alpha) \mathrm{e}^{-r}\right]\left(1-r^{2}\right)-2 r=0, \quad r \in(0,1) .
$$

Corollary 6. Let $\theta \in \mathcal{A}, \Theta \in \mathcal{S} \mathcal{L}^{*}(\alpha)$ with $\theta(z) \ll \Theta(z)$. Then for $|z| \leq r_{7}$, we get $\left|\theta^{\prime}(z)\right| \leq\left|\Theta^{\prime}(z)\right|$, where $r_{7}$ is the smallest positive root of the equation

$$
[\alpha+(1-\alpha) \sqrt{1-r}]\left(1-r^{2}\right)-2 r=0, \quad r \in(0,1)
$$

The following result concludes a majorization property for a subset $\mathcal{S}_{R L}^{*}=\mathcal{S}^{*}\left(\varphi_{0}\right)$ introduced by Mendiratta et al. considering Theorem 2.2 in [25], in which

$$
\varphi_{0}(z)=\sqrt{2}-j \sqrt{\frac{1-z}{1+2 j z}} \quad j=\sqrt{2}-1,
$$

where function $\varphi_{0}$ is a univalent and convex in $\mathbb{U}$.

Corollary 7. Let $\theta \in \mathcal{A}, \Theta \in \mathcal{S}_{R L}^{*}$ with $\theta(z) \ll \Theta(z)$. Then $\left|\theta^{\prime}(z)\right| \leq\left|\Theta^{\prime}(z)\right|$ for $|z| \leq r_{8}$, where $r_{8}$ is the smallest positive root of the equation

$$
\varphi_{0}(r)\left(1-r^{2}\right)-2 r=0, \quad r \in(0,1) .
$$


In the following result, we get a majorization property for a category $\mathcal{S}^{*}\left(p_{l}(z)\right)$ introduced by Kanas and Wiśniowska in [26] in which

$$
p_{l}(z)=1+P_{1}(l) z+P_{2}(l) z^{2}+\cdots,
$$

where $p_{k}(z)$ satisfies the conclusion of Remark 1 (see also $[27,28]$ ).

Corollary 8. Let $\theta \in \mathcal{A}, \Theta \in \mathcal{S}^{*}\left(p_{k}(z)\right)$ with $\theta(z) \ll \Theta(z)$. Then for $|z| \leq r_{9}$, we have $\left|\theta^{\prime}(z)\right| \leq\left|\Theta^{\prime}(z)\right|$, where $r_{9}$ is the smallest positive root of the equation

$$
p_{l}(-r)\left(1-r^{2}\right)-2 r=0, \quad r \in(0,1) .
$$

Since $\vartheta(z)=(1+C z) /(1+D z)$ satisfies in Remark 1 we obtain a majorization property for the class $\mathcal{S}^{*}[C, D]$ as follows:

Corollary 9. Let $\theta \in \mathcal{A}, \Theta \in \mathcal{S}^{*}[C, D]$ with $\theta(z) \ll \Theta(z)$. Then for $|z| \leq r_{10}$, we get $\left|\theta^{\prime}(z)\right| \leq\left|\Theta^{\prime}(z)\right|$, where $r_{10}$ is the smallest positive root of the equation

$$
(1-C)\left(1-r^{2}\right)-2 r(1-D)=0, \quad r \in(0,1) .
$$

To prove the following result, we state the next lemma due to Kuroki and Owa [29] (see also [30]).

Lemma 2. Let $\vartheta$ be a convex in $\mathbb{U}$ with form $\vartheta(z)=1+\sum_{n=1}^{\infty} B_{n} z^{n}$. If $f \in \mathcal{S}^{*}(\vartheta)$, then

$$
\left|a_{n}\right| \leq \frac{\prod_{m=2}^{n}\left(m-2+\left|B_{1}\right|\right)}{(n-1) !} \quad(n=2,3, \cdots) .
$$

Theorem 3. Let $\vartheta$ be convex in $\mathbb{U}$ and $\theta(z)=z+\sum_{n=2}^{\infty} a_{n} z^{n} \in \mathcal{A}, \Theta(z)=z+\sum_{n=2}^{\infty} b_{n} z^{n} \in \mathcal{S}^{*}(\vartheta)$ with $\theta(z) \ll \Theta(z)$. Then

$$
\left|a_{n}\right| \leq 1+\sum_{j=2}^{n}\left(\frac{\prod_{m=2}^{j}\left(m-2+\left|B_{1}\right|\right)}{(j-1) !}\right) \quad(n=2,3, \cdots) .
$$

Proof. Since $\theta(z) \ll \Theta(z)$, by the majorization principle there is an analytic function $v(z)=\sum_{n=0}^{\infty} c_{n} z^{n}$ with $|v(z)| \leq 1$ satisfying

$$
\theta(z)=v(z) \Theta(z)
$$

where it concludes,

$$
a_{n}=c_{0} b_{n}+c_{1} b_{n-1}+\cdots+c_{n-2} b_{2}+c_{n-1} .
$$

If $\gamma$ is any circle $|z|=r, 0<r<1$, where $z=r \mathrm{e}^{i \zeta}, 0 \leq \zeta \leq 2 \pi$, then

$$
c_{k}=\frac{1}{2 \pi \mathrm{i}} \int_{\gamma} \frac{v(z)}{z^{k+1}} \mathrm{~d} z \quad \text { for } \quad k=0,1, \cdots, n-1 .
$$

In view of the above equality, we can write the equality (7) in the form (see [4], p. 99)

$$
a_{n}=\frac{1}{2 \pi \mathrm{i}} \int_{\gamma} \frac{v(z)}{z^{n}}\left[1+b_{2} z+\cdots+b_{n} z^{n-1}\right] \mathrm{d} z .
$$


From the above equality for $n \geq 2$, we obtain

$$
\begin{aligned}
\left|a_{n}\right| & \leq \frac{1}{2 \pi} \int_{0}^{2 \pi} \frac{1}{r^{n-1}}\left|1+b_{2} r \mathrm{e}^{i \zeta}+\cdots+b_{n} r^{n-1} \mathrm{e}^{i(n-1) \zeta}\right| \mathrm{d} \zeta \\
& \leq \frac{1}{r^{n-1}}\left(1+\left|b_{2}\right|+\cdots+\left|b_{n}\right|\right) .
\end{aligned}
$$

Since this inequality holds for all $r$ in the interval $0<r<1$, it follows that

$$
\left|a_{n}\right| \leq 1+\left|b_{2}\right|+\cdots+\left|b_{n}\right| .
$$

Now using Lemma 2 we have

$$
\left|a_{n}\right| \leq 1+\sum_{j=2}^{n}\left(\frac{\prod_{m=2}^{j}\left(m-2+\left|B_{1}\right|\right)}{(j-1) !}\right)
$$

which completes the proof.

Corollary 10. Let $\theta(z)=z+\sum_{n=2}^{\infty} a_{n} z^{n} \in \mathcal{A}, \Theta(z)=z+\sum_{n=2}^{\infty} b_{n} z^{n} \in \mathcal{S} \mathcal{T}_{\text {hpl }}(b)$ with $\theta(z) \ll \Theta(z)$. Then

$$
\left|a_{n}\right| \leq 1+\sum_{j=2}^{n}\left(\frac{\prod_{m=2}^{j}(m+b-2)}{(j-1) !}\right) \quad(n=2,3, \cdots) .
$$

Corollary 11. Let $\theta(z)=z+\sum_{n=2}^{\infty} a_{n} z^{n} \in \mathcal{A}, \Theta(z)=z+\sum_{n=2}^{\infty} b_{n} z^{n} \in \mathcal{S}^{*}\left(\mathrm{e}^{z}\right)$ with $\theta(z) \ll \Theta(z)$. Then

$$
\left|a_{n}\right| \leq 1+\sum_{j=2}^{n}\left(\frac{\prod_{m=2}^{j}(m-1)}{(j-1) !}\right)=n \quad(n=2,3, \cdots) .
$$

Since the identity function $\Theta(z)=z$ belongs to the category $\mathcal{S}^{*}\left(\mathrm{e}^{z}\right)$, from Corollary 11 we get the next result:

Example 3. Let $\theta \in \mathcal{A}$ and $|\theta(z)|<1$, then

$$
\left|a_{n}\right| \leq n \quad(n=2,3, \cdots) .
$$

\section{Conclusions}

In the current paper, we obtain a majorization result for a general category $\mathcal{S}^{*}(\vartheta)$ of starlike functions. Also, we investigate coefficient bounds for majorized functions associated with the class $\mathcal{S}^{*}(\vartheta)$. Furthermore, we can consider some particular functions $\vartheta$ in Theorems 2 and 3 to get the corresponding majorization results.

Author Contributions: Investigation, N.E.C., Z.O., E.A.A. and A.E. All authors have read and agreed to the published version of the manuscript.

Funding: The first author was supported by the Basic Science Research Program through the National Research Foundation of Korea (NRF) funded by the Ministry of Education, Science and Technology (No. 2019R111A3A01050861).

Conflicts of Interest: The authors declare no conflict of interest. 


\section{References}

1. Robertson, M.S. Quasi-subordinate functions. In Mathematical Essays Dedicated to A. J. MacIntyre; Ohio University Press: Athens, OH, USA, 1970; pp. 311-330.

2. Robertson, M.S. Quasi-subordination and coefficient conjecture. Bull. Am. Math. Soc. 1970, 76, 1-9. [CrossRef]

3. Duren, P.L. Univalent Functions; Grundlehren der Mathematischen Wissenschaften, Band 259; Springer: New York, NY, USA; Berlin/Heidelberg, Germany; Tokyo, Japan, 1983.

4. MacGreogor, T.H. Majorization by univalent functions. Duke Math. J. 1967, 34, 95-102. [CrossRef]

5. Ma, W.C.; Minda, D. A unified treatment of some special classes of univalent functions. In Proceedings of the Conference on Complex Analysis (Tianjin, 1992); International Press: Cambridge, MA, USA, 1992; pp. 157-169.

6. Sokól, J.; Stankiewicz, J. Radius of convexity of some subclasses of strongly starlike functions. Zesz. Nauk. Rzesz. Matematyka. 1996, 19, 101-105.

7. Mendiratta, R.; Nagpal, S.; Ravichandran, V. On a subclass of strongly starlike functions associated with exponential function. Bull. Malays. Math. Sci. Soc. 2015, 38, 365-386. [CrossRef]

8. Raina, R.K.; Sokół, J. Some properties related to a certain class of starlike functions. Comptes Rendus Mathematique 2015, 353, 973-978. [CrossRef]

9. Kanas, S.; Masih, V.S.; Ebadian, A. Relations of a planar domains bounded by hyperbolas with families of holomorphic functions. J. Inequal. Appl. 2019, 2019, 246. [CrossRef]

10. Goel, P.; Sivaprasad Kumar, S. Certain class of starlike functions associated with modified sigmoid function. Bull. Malays. Math. Sci. Soc. 2020, 43, 957-991. [CrossRef]

11. Altintas, O.; Özkan, Ö.; Srivastava, H.M. Majorization by starlike functions of complex order. Complex Var. Theory Appl. 2001, 46, 207-218. [CrossRef]

12. Altintas, O.; Srivastava, H.M. Some majorization problems associated with p-valently starlike and convex functions of complex order. East Asian Math. J. 2001, 17, 207-218.

13. Carathéodory, C. Theory of Functions of a Complex Variable; Chelsea Publishing Company: New York, NY, USA, 1954; Volume 2.

14. Goswami, P.; Aouf, M.K. Majorization properties for certain classes of analytic functions using the Salagean operator. Appl. Math. Lett. 2010, 23, 1351-1354. [CrossRef]

15. Goyal, S.P.; Goswami, P. Majorization for certain classes of analytic functions defined by fractional derivatives. Appl. Math. Lett. 2009, 22, 1855-1858. [CrossRef]

16. Goyal, S.P.; Goswami, P. Majorization for certain classes of meromorphic functions defined by integral operator. Ann. Univ. Mariae Curie Sklodowska Lub.-Pol. 2012, 2, 57-62. [CrossRef]

17. Li, S.-H.; Tang, H.; Ao, E. Majorization properties for certain new classes of analytic functions using the Salagean operator. J. Inequal. Appl. 2013, 2013, 86. [CrossRef]

18. Panigraht, T.; El-Ashwah, R. Majorization for subclasses of multivalent meromorphic functions defined through iterations and combinations of the Liu-Srivastava operator and a meromorphic analogue of the Cho-Kwon-Srivastava operator. Filomat 2017, 31, 6357-6365. [CrossRef]

19. Prajapat, J.K.; Aouf, M.K. Majorization problem for certain class of p-valently analytic functions defined by generalized fractional differintegral operator. Comput. Math. Appl. 2012, 63, 42-47. [CrossRef]

20. Tang, H.; Aouf, M.K.; Deng, G. Majorization problems for certain subclasses of meromorphic multivalent functions associated with the Liu-Srivastava operator. Filomat 2015, 29, 763-772. [CrossRef]

21. Tang, H.; Srivastava, H.M.; Li, S.-H.; Deng, G.-T. Majorization results for subclasses of starlike functions based on the sine and cosine functions. Bull. Iran Math. Soc. 2019. [CrossRef]

22. Kanas, S.; Sugawa, T. On conformal representations of the interior of an ellipse. Ann. Acad. Sci. Fenn. Math. 2006, 31, 329-348.

23. Cho, N.E.; Kumar,V.; Kumar, S.S.; Ravichandran, V. Radius problems for starlike functions associated with the sine function. Bull. Iran. Math. Soc. 2019, 45, 213-232. [CrossRef]

24. Khatter, K.; Ravichandran, V.; Kumar, S.S. Starlike functions associated with exponential function and the lemniscate of Bernoulli. Rev. Real Acad. Cienc. Exactas Físicas Nat. Ser. A Mat. 2019, 113, 233-253. [CrossRef]

25. Mendiratta, R.; Nagpal, S.; Ravichandran, V. A subclass of starlike functions associated with left-half of the lemniscate of Bernoulli. Int. J. Math. 2014, 25, 1450090. [CrossRef]

26. Kanas, S.; Wiśniowska, A. Conic domains and starlike functions. Rev. Roum. Math. Pure. Appl. 2000, 45, 647-658. 
27. Kanas, S. Coefficient estimates in subclasses of the Caratheodory class related to conical domains. Acta Math. Univ. Comen. 2005,74, 149-161.

28. Kanas, S.; Wiśniowska, A. Conic regions and k-uniform convexity. J. Comput. Appl. Math. 1999, 105, $327-336$. [CrossRef]

29. Kuroki, K.; Owa, S. Notes on new class for certain analytic functions. RIMS Kokyuroku 2011, 1772, 21-25.

30. Xu, Q.H.; Gui, Y.C.; Srivastava, H.M. Coefficient estimates for certain subclasses of analytic functions of complex order. Taiwanese J. Math. 2011, 15, 2377-2386. [CrossRef]

(C) 2020 by the authors. Licensee MDPI, Basel, Switzerland. This article is an open access article distributed under the terms and conditions of the Creative Commons Attribution (CC BY) license (http:/ / creativecommons.org/licenses/by/4.0/). 